\title{
El test de Bender y las dificultades de aprendizaje en matemática de los estudiantes con necesidades especiales de la ciudad de Puno, Perú
}

Bender test and learning difficulties in mathematics of students with special needs of the Puno city, Peru

\section{Volumen 17, Número 3 \\ Setiembre-Diciembre}

pp. 1-16

\author{
Heber Nehemias Chui Betancur \\ Percy Samuel Yabar Miranda \\ Silvia Verónica Valdivia Yabar \\ Sara María Arista Santisteban \\ Revista indizada en REDALYC, SCIELO
}

Revista distribuida en las bases de datos:

\section{LATINDEX, DOAJ, REDIB, IRESIE, CLASE, DIALNET, SHERPA/ROMEO,} QUALIS-CAPES, MIAR

Revista registrada en los directorios:

ULRICH'S $, \underline{R E D I E}, \underline{\text { RINACE}}, \underline{\text { OEI }}$ MAESTROTECA, PREAL, $\underline{\text { CLACSO }}$ 


\title{
El test de Bender y las dificultades de aprendizaje en matemática de los estudiantes con necesidades especiales de la ciudad de Puno, Perú
}

Bender test and learning difficulties in mathematics of students with special needs of the

Puno city, Peru

\author{
Heber Nehemias Chui Betancur ${ }^{1}$ \\ Percy Samuel Yabar Miranda² \\ Silvia Verónica Valdivia Yabar ${ }^{3}$ \\ Sara María Arista Santisteban ${ }^{4}$
}

\begin{abstract}
Resumen: El presente artículo abordó el grado de correlación entre el nivel de aprendizaje y la maduración visomotriz en el aprendizaje de los estudiantes con necesidades especiales de las Instituciones Educativas Niño Jesús de Praga y Nuestra Señora de Copacabana de la ciudad de Puno, a fin de comprobar el aporte predictivo de la edad de maduración visomotora en el aprendizaje de la matemática. Se aplicó una ficha de análisis documental de los niveles de aprendizaje en matemática y el denominado test de Bender, esto para evaluar la edad de maduración visomotriz a una muestra compuesta por 38 estudiantes. Los resultados muestran que 22 estudiantes de la primera Institución Educativa tienen una edad cronológica de 12,70 años en promedio $(D E=3,71$ años), y la edad promedio de maduración visomotriz para el aprendizaje es igual a 4 años (DE=0,55 años). Asimismo, se mostró que 16 estudiantes de la segunda Institución Educativa tienen una edad cronológica de 12,83 años en promedio ( $D E=3,00$ años), y la edad promedio de maduración visomotriz para el aprendizaje es igual a 3,68 años ( $D E=0,60$ años). Estos resultados evidencian la existencia de una correlación $r=0,70$ positiva y significativa entre los niveles de aprendizaje en matemática y la edad de maduración visomotriz para el aprendizaje, esto significa que los niveles de aprendizaje de la matemática dependen de la maduración visomotora. Consecuentemente es necesario implementar el cálculo de la edad de maduración en la diversificación curricular para que los servicios educativos sean adecuados y pertinentes para los estudiantes con necesidades especiales.
\end{abstract}

Palabras clave: aprendizaje, matemática, madurez, percepción visomotriz.

Abstract: The present paper addressed the degree of correlation between the level of learning and visual maturation maturation for the learning of the children with special needs of the Educational Institutions Niño Jesús de Praga and Our Lady of Copacabana of the city of Puno to verify the contribution Predictive of the age of visomotor maturation in the learning of mathematics. A documentary analysis of learning levels in mathematics and the Bender test were applied to evaluate the age of visual-maturation maturation to a sample composed of 38 students. The results show that 22 students of the first Educational Institution have a chronological age of 12.70 years on average $(S D=3.71$ years) and the average age of visual-maturation maturation for learning is equal to 4 years $(S D=0,55$ years). Likewise, 16 students of the second Educational Institution have a chronological age of 12.83 years on average ( $S D=3.00$ years) and the average age of visual-maturation for learning is equal to 3.68 years on average $(D E=0.60$ years). These results show the existence of a positive and significant $r=0.70$ correlation between learning levels in mathematics and the age of visual-maturation maturation for learning, which means that the learning levels of mathematics depend on visual-maturation maturation. Consequently, it demands to implement the calculation of the age of maturation in the curricular diversification in such a way that the educational services are suitable and pertinent for the children with special needs.

Keywords: learning, mathematic, maturity, visual-motor perception.

\footnotetext{
${ }_{1}^{1}$ Universidad Nacional del Altiplano, Puno, Perú. Catedrático. Dirección electrónica: heberchui@gmail.com

2 Universidad Nacional del Altiplano, Puno, Perú. Catedrático. Dirección electrónica: Cyrpe368@hotmail.com

${ }^{3}$ Universidad Nacional Autónoma de México. Catedrática Visitante. Dirección electrónica: siveroval@gmail.com

${ }^{4}$ Universidad Nacional del Altiplano, Puno,Perú.Catedrática.Dirección electrónica: Sara.arista.s@gmail.com
}

Artículo recibido: 4 de octubre, 2016

Enviado a corrección: 27 de abril, 2017

Aprobado: 5 de junio, 2017 


\section{Introducción}

El Test Gestáltico de Bender (TGB) ha tenido una larga historia de evaluación psicológica, a partir de ello se han desarrollado varios sistemas de calificación, uno de ellos es el sistema de puntuación que evalúa la edad de maduración visomotriz del aprendizaje (Koppitz, 1981; Merino, 2014). La maduración es un factor determinante que influye en el aprendizaje, y es un proceso natural, su secuencia está predeterminada biológicamente (Bedard y Dhuey, 2006). La maduración facilita el proceso de aprendizaje y se hace efectivo cuando la madurez apropiada ha sido alcanzada (Suehiro y Santos, 2006).

El aprendizaje se lleva a cabo solo si el escenario se ha logrado a través de un proceso de maduración. En los años treinta, se dieron a conocer los múltiples usos y aplicaciones del test de Bender con enfoque pedagógico en particular el sistema de evaluación de Kopitz, el cual se utiliza en diferentes países de todo el mundo (Rinaldi y Bocato, 2012). El test de Bender es un test que demanda de los niños y niñas copiar diseños conocidos internacionalmente, y cuyo objetivo es evaluar la madurez perceptiva, un deterioro neurológico y problema emocional (Sisto, Bartholomeu, Rueda, Santos y Noronha, 2008). Según Koppitz (1989), la mayoría de la población infantil con problemas emocionales y de conducta evidencian problemas de aprendizaje, por lo que su desempeño en el test de Bender es mínimo.

En la presente investigación se han identificado los niveles de aprendizaje en matemática y los niveles de madurez de la niñez con necesidades especiales de las instituciones educativas especiales Educativas Niño Jesús de Praga y Nuestra Señora de Copacabana de la ciudad de Puno, para proponer que, en la diversificación curricular, se realicen las adaptaciones curriculares y la selección de estrategias metodológicas acordes con la edad de maduración, de manera que se ofrezca un servicio pertinente y de calidad a uno de los grupos minoritarios de nuestro país. Los resultados evidencian la existencia de una correlación $r=0,70$, positiva y significativa entre los niveles de aprendizaje en matemática y la edad de maduración visomotriz para el aprendizaje, lo cual significa que los niveles de aprendizaje de la matemática dependen de la maduración visomotora. Consecuentemente, resulta prioritario implementar el cálculo de la edad de maduración de la niñez en la diversificación curricular de educación primaria, de tal manera que las escuelas sean más inclusivas. 


\section{Referente teórico}

La maduración de la percepción visomotriz es el desenvolvimiento en la percepción y reproducción de las figuras Gestálticas, no provocadas por el ejercicio ni por la experiencia, que están determinadas por principios biológicos de acción sensoriomotriz, (Keller, 1955; Koppitz, 1962). Según Chan, (2000) la maduración de la percepción visomotriz es el proceso mediante el cual el individuo alcanza su máximo desarrollo y puede evidenciarse en la reproducción correcta de las nueve figuras del test de Bender. Para Allport (1961), la maduración es el proceso fisiológico, genéticamente determinado, por el cual un órgano o conjunto de órganos llega a una madurez y permite la función por la cual es conocido.

Como señalan Bedard y Dhuey (2006), en su estudio, La persistencia de la madurez de la primera infancia: Evidencia Internacional de edad efectos largo plazo, "La maduración es un factor muy importante que influye en el aprendizaje, y es un proceso natural, su secuencia está predeterminada biológicamente" (p. 1441).

La maduración, por lo tanto, no depende de la edad cronológica del niño o niña, sino que depende de factores intrínsecos que determinan el momento y forma de aparición de actividades motoras específicas. En el presente artículo se considera la definición de maduración según Koppitz. Como se mencionó, en la función Gestáltica Visomotora intervienen una serie de funciones que van cambiando a medida que la persona madura. No existe una ley en cuanto a la maduración, de tal suerte que un sujeto puede madurar más rápido en algunos aspectos y de forma más lenta que otros. Esta variable fue la que consideró la Dra. Koppitz para construir su escala denominada "Escala de Maduración para el Bender Infantil". Este Sistema busca detectar en la niñez madurez para el aprendizaje, problemas en la lectura, dificultades emocionales, lesión cerebral y deficiencia mental (Koppitz, 1962).

En el mundo en que vivimos, la matemática está presente en diversas manifestaciones de la cultura y la naturaleza. Como lo señala el Informe del Programa Internacional para la Evaluación de Estudiantes (PISA) (2012), en nuestro alrededor, podemos encontrar una amplia gama de fenómenos visuales y físicos, propiedades de los objetos, posiciones y direcciones, representaciones de los objetos, su codificación y decodificación. La presente investigación se centra básicamente en conocer los niveles de madurez para el aprendizaje y los niveles de aprendizaje en matemática que permiten comparar e identificar las capacidades y competencias, y finalmente establecer el grado de correlación entre los 
niveles de aprendizaje en matemática y la maduración de la percepción visomotriz de la población infantil de instituciones educativas especiales de la ciudad de Puno, Perú.

Según el Ministerio de Educación de Perú Minedu (2013), los niveles de logro se pueden medir en cuatro categorías, las cuales se detallan a continuación: Logro Desatacado [18-20 puntos], consiste en que la persona estudiante evidencia el logro de los aprendizajes previstos, a través de la demostración de un manejo solvente y muy satisfactorio en todas las tareas propuestas. El estudiantado que alcanza este nivel, según PISA (2012), puede aplicar sus conocimientos y destrezas al establecer relaciones entre la forma y figura geométrica, desarrolla procedimientos como medir y comparar longitudes de los objetos de su entorno en dos y tres dimensiones, para enfrentar situaciones novedosas. Logro Previsto [14-17 puntos] en esta categoría el estudiante evidencia el logro de los aprendizajes previstos en el tiempo programado, las personas que se sitúan en este nivel, según PISA (2012), son capaces de ejecutar procedimientos descritos claramente con la medición de las figuras geométricas, además compara longitudes de los objetos de su entorno en dos y tres dimensiones, y establece relación entre la forma y figura geométrica, asociándolos directamente a situaciones del mundo real. En Proceso [11-13 puntos] En esta categoría el estudiante está en camino de lograr los aprendizajes previstos, para lograrlo requiere acompañamiento durante un tiempo razonable, es decir, según el informe PISA (2012), los y las estudiantes en este nivel son capaces de establecer relaciones entre la forma y figura geométrica, desarrollando procedimientos como medir y comparar longitudes de los objetos de su entorno en dos y tres dimensiones a través de procedimientos rutinarios conforme a instrucciones directas en situaciones explícitas. En Inicio [0-10] En esta categoría el estudiante está empezando a desarrollar los aprendizajes previstos o evidencia dificultades para el desarrollo de estos, y necesita mayor tiempo de acompañamiento e intervención del docente de acuerdo con su ritmo y estilo de aprendizaje, esto significa que se trata de estudiantes que no son capaces de realizar las tareas como: establecer relaciones entre la forma y figura geométrica, ni medir ni comparar longitudes de los objetos de su entorno en dos y tres dimensiones más elementales, el cual evidencia la categoría en inicio según PISA (2012).

\section{Materiales y métodos}

El Sistema de Koppitz, publicado en 1964, busca detectar en la niñez con necesidades especiales, la madurez para el aprendizaje, problemas en la lectura, dificultades 
emocionales, lesión cerebral y deficiencia mental. La validación de la escala inicial se realizó con la Prueba de Maduración Escolar Metropolitana ( $n=165$ estudiantes, de seis escuelas con diferente nivel sociocultural), con una validez interjueces del 93\% (Koppitz, 1981). El sistema de evaluación de la Prueba de Bender elaborado por la Dra. Koppitz fue desarrollado a partir de una revisión de los diferentes métodos de calificación entonces utilizados.

Así, en 1958 comienza la preparación de una prueba breve para valorar la percepción visomotriz en niñas y niños, para lo cual se confeccionó una escala de maduración con 20 categorías de distorsión iniciales: distorsión de la forma, rotación, borraduras, omisiones, orden confuso, superposición de las figuras, comprensión, segundo intento, perseveración, círculos o rayas en lugar de puntos, línea ondulada, forma de los círculos, desviación en la oblicuidad, rayas o puntos en lugar de círculos, achatamiento, número incorrecto de puntos, desunión entre cuadrado y la curva, ángulos en las curvas, omisión o adición de ángulos y recuadros. Con base en estos factores, se realizaron análisis de $\mathrm{Chi}^{2}$ entre una población de 1200 estudiantes de Ohio, de entre 5 y 10 años de edad, con diferente nivel de inteligencia, funcionamiento neurológico y ajuste emocional, con un valor a $=0.89$ (Koppitz, 1981). La aplicación del sistema de evaluación de Kopitz para el test de Bender, para niñas y niños, ha permanecido muy popular a través de los años y ha generado más de 300 artículos científicos publicados en diversas revistas del mundo (Reynolds, 2007).

Se utilizó el Test de Bender - Koppitz para evaluar la madurez del aprendizaje de las niñas y los niños de 2 instituciones educativas especiales (Niño Jesús de Praga y Virgen de Copacabana), la aplicación del test de Bender consistió en pedirle a las niñas y a los niños que copiaran las 9 figuras (Gestalt) para luego analizarlas y evaluarlas (En la investigación se utilizó un nivel de significancia de 0,05 y para presentar los resultados se utilizó un programa estadístico SPSS, de la versión 11,5) en las categorías de distorsión de la forma, rotación, integración y perseveración (Tendencia a mantener y a repetir el mismo tipo de respuesta ante preguntas, situaciones o estímulos diferentes), con la finalidad de conocer la edad de madurez visomotora para el aprendizaje que manifiesta el estudiantado de instituciones educativas especiales de la ciudad de Puno, cuyo instrumento es la ficha del Test de Bender - Koppitz, seguidamente se calculó la edad del aprendizaje a fin de comparar con la escala que Koppitz propuso. 


\subsection{El test de Bender - Koppitz}

El aplicador les proporcionó a los estudiantes: las tarjetas gestálticas de Bender (cuyos nombres están definidas como: tarjeta $A$, tarjeta 1 , tarjeta 2 , tarjeta 3 , tarjeta 4 , tarjeta 5 , tarjeta 6, tarjeta 7 y tarjeta 8 tal como se puede apreciar en la figura 1), lápiz, borrador, tajador de lápices o afilador de lápices, para que ellos copien de la manera más similar posible.

Se presentó las niñas y niños una a una cada tarjeta, seguidamente las niñas y niños preguntaron: ¿Puedo copiar las figuras en una sola hoja? ¿Puedo copiar una figura en cada hoja?, ante mencionadas preguntas el aplicador dijo frases neutrales como: "copia la figura de la manera más parecida que puedas", además se les proporcionó todas las hojas que los participantes pidieron, respecto al tiempo de aplicación, se esperó a que todos terminaran de copiar los diseños si necesidad de apurarlos.

Figura 1

Las tarjetas gestálticas de Bender

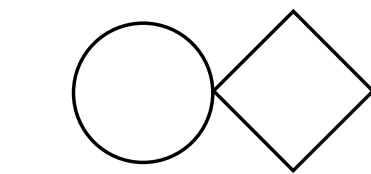

Tarjeta A

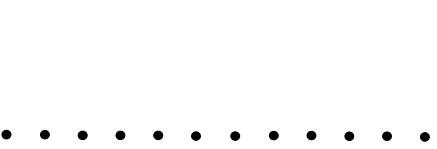

Tarjeta 1

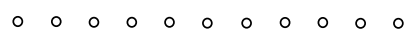

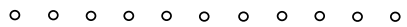

Tarjeta 2
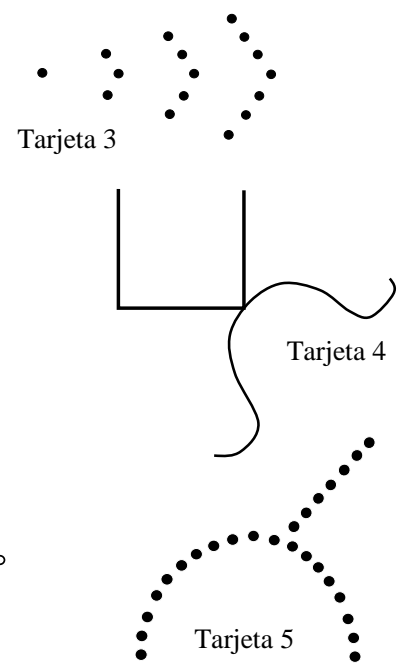

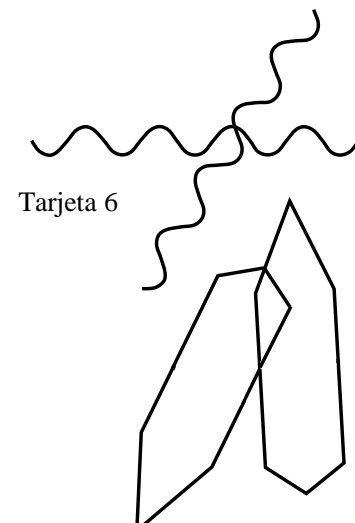

Tarjeta 7

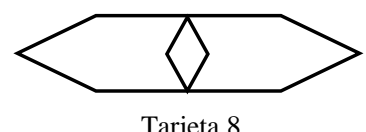

Fuente: Bender (1938)

Para calificar las tarjetas de Bender se utilizó el sistema de calificación de Koppitz, en el que se asigna puntaje de uno o cero, se le asigna cero si hay ausencia de error y se le asigna uno cuando se observa el error. Tarjeta $A$ (ver Figura 1) Las dimensiones Distorsión de la forma, Rotación e Integración se califican de la siguiente manera: en distorsión de la 
forma, se puntúa con uno en dos casos; primero, cuando el cuadrado, el círculo o ambos están excesivamente achatados o deformados; segundo, cuando existe la desproporción entre el tamaño del cuadrado y el del círculo (uno es el doble de grande que el otro). En rotación, se puntúa con uno cuando se aprecia la rotación de la figura o parte de esta en más de 45․ En integración, se puntúa con uno cuando se aprecia la falla en el intento de unir el círculo y el cuadrado; el círculo y el vértice adyacente del cuadrado se encuentran separados o superpuestos en más de $3 \mathrm{~mm}$.

En la tarjeta 1 (ver Figura 1), se califican las dimensiones Distorsión de la forma, Rotación y Perseveración de la siguiente manera: en distorsión de la forma, se puntúa con uno cuando cinco o menos puntos de la figura son convertidos en círculos, cuando los puntos son agrandados o círculos parcialmente llenados. En rotación, se puntúa con uno cuando la figura es rotada en $45^{\circ}$ o más. En Perseveración se puntúa con uno cuando se realizan más de 15 puntos en una hilera.

En la tarjeta 2 (ver Figura 1), se califican las dimensiones Rotación, Integración y Perseveración de la siguiente manera: En rotación, se puntúa con uno, cuando la rotación de la figura es en 45o o más. En Integración se puntúa con uno cuando se observa la omisión de una o más hileras de círculos. En Perseveración se puntúa con uno cuando se realizan más de 14 columnas de círculos en una hilera.

En la tarjeta 3 (ver Figura 1), se califican las dimensiones Distorsión de la forma, Rotación e Integración, de la siguiente manera: En distorsión de la forma, se puntúa con uno cuando cinco o más puntos de la figura original son copiados con círculos, puntos agrandados o círculos parcialmente rellenos en lugar de puntos. En rotación, se puntúa uno cuando la figura copiada es rotada en $45^{\circ}$ o más. En integración, se puntúa con uno en dos casos: primero, cuando la figura copiada evidencia la desintegración del diseño: aumento de cada hilera sucesiva de puntos no lograda, "cabeza de flecha" irreconocible o invertida, conglomeración de puntos. Segundo, cuando en la figura copiada se reemplaza por una o más líneas en lugar de hilera de punto; la línea puede sustituir a los puntos o estar agregada a estos.

En la tarjeta 4 (ver Figura 1), se califican las dimensiones Rotación e Integración de la siguiente manera: En rotación, se puntúa con uno cuando la figura copiada está rotada en 45o o más. En Integración, se puntúa con uno cuando se observa en la figura copiada una separación de $3 \mathrm{~mm}$ entre la curva y el ángulo adyacente. 
En la tarjeta 5 (ver Figura 1), se califican las dimensiones Distorsión de la forma, Rotación e Integración de la siguiente manera: En distorsión de la forma, se puntúa con uno cuando cinco o más puntos de la tarjeta original es reemplazada por círculos o puntos agrandados. En rotación, se puntúa con uno cuando la figura esta rotada en $45^{\circ}$ o más; rotación de la extensión (apunta hacia la derecha o la izquierda). En integración, se evalúa en dos casos: primero, se puntúa con uno cuando la figura copiada evidencia la desintegración del diseño, conglomeración de puntos, línea recta o círculo de puntos en lugar de arco. Segundo, se puntúa con uno cuando en la figura copiada por el niño o niña, es reemplazada por línea continua en lugar de una hilera de puntos ya sea en el arco y/o la extensión.

En la tarjeta 6 (ver Figura 1), se califican las dimensiones Distorsión de la forma, Integración y Perseveración de la siguiente manera: En distorsión de la forma se evalúa en dos casos: Primero, se puntúa con uno, cuando tres o más curvas son sustituidas por ángulos (como los dientes de un serrucho). Segundo se puntúa con uno cuando una o ambas líneas curvadas de la tarjeta original son sustituidas por líneas rectas. En integración, se puntúa con uno cuando las dos líneas no se cruzan o se cruzan en el extremo de una o de ambas líneas, dos líneas onduladas entrelazadas. En Perseveración, se puntúa con uno cuando en la figura copiada, se muestran seis o más curvas sinusoidales completas en cualquiera de las dos direcciones.

En la tarjeta 7 (ver Figura 1), se califican las dimensiones: Distorsión de la forma, Rotación e Integración, de la siguiente manera: En distorsión de la forma, se evalúan dos casos: primero se puntúa con uno cuando en la figura copiada existe desproporción entre el tamaño de los 2 hexágonos (para puntuar con uno, un hexágono debe ser al menos el doble de grande que el otro). Segundo, se puntúa con uno, cuando los hexágonos copiados están excesivamente deformados o presente adición u omisión de ángulos. En rotación se puntúa con uno cuando la figura copiada presenta rotación en $45^{\circ}$ o más. En integración se puntúa con uno cuando los hexágonos copiados no se superponen o lo hacen excesivamente.

En la tarjeta 8 (ver Figura 1), se evalúan las dimensiones Distorsión de la forma y Rotación de la siguiente manera: En distorsión de la forma, se puntúa con uno cuando el hexágono o el rombo copiado se presentan excesivamente deformados o cuando se agregan u omiten los ángulos de la figura original. En rotación se puntúa con uno cuando la figura copiada, esta rotada en $45^{\circ} 0$ más. 


\subsection{Participantes}

En la presente investigación se seleccionó la muestra a conveniencia, debido a que la investigación exigió trabajar con una población infantil que requiere atención especial. La muestra de estudio estuvo constituida por la población infantil de la Institución Educativa Niño Jesús de Praga y la Institución Educativa Nuestra Señora de Copacabana, ambas localizadas en la ciudad de Puno, la investigación se realizó en el año 2014, que se detalla en el tabla 1.

Tabla 1

Muestra de estudiantes de las instituciones educativas especiales del nivel primario de la ciudad de Puno

\begin{tabular}{lcccc}
\hline & \multicolumn{3}{c}{$\begin{array}{c}\text { Muestra (Según edad } \\
\text { cronológica) }\end{array}$} & \\
\cline { 2 - 4 } \multicolumn{1}{c}{ INSTITUCIÓN EDUCATIVA } & $\begin{array}{l}\text { Menos } \\
\text { de } 10 \\
\text { años }\end{array}$ & $\begin{array}{l}\text { Entre } \\
10 \text { y } 14 \\
\text { años }\end{array}$ & $\begin{array}{c}\text { Más de } \\
14 \text { años }\end{array}$ & $\begin{array}{c}\text { SUB } \\
\text { TOTAL }\end{array}$ \\
\hline $\begin{array}{l}\text { Institución Educativa de } \\
\begin{array}{l}\text { educación especial Niño Jesús de } \\
\text { Praga. }\end{array}\end{array}$ & 5 & 9 & 8 & 22 \\
\hline $\begin{array}{l}\text { Institución Educativa de } \\
\text { educación especial Nuestra }\end{array}$ & 3 & 7 & 6 & 16 \\
$\begin{array}{l}\text { Señora de Copacabana } \\
\text { TOTAL }\end{array}$ & 8 & 16 & 14 & 38 \\
\hline
\end{tabular}

Fuente: Actas consolidadas de las Instituciones educativas especiales de la ciudad de Puno en el año 2014

La Institución Educativa Niño Jesús de Praga, ubicada en la Av. Ejército № 670 Urbanización Chanu Chanu I Etapa en el distrito de Puno, fue creada con RD № 620 DREP, con autorización de funcionamiento RD № 0355-77-VII-DRE, con tipo de gestión pública correspondiente a la UGEL-Puno,. Dicha institución presta servicios a niñas y niños con necesidades especiales con diagnóstico moderado y severo, en la modalidad escolarizado en los niveles de educación inicial y educación primaria. La Escuela cuenta con docentes especializados en atención a la niñez con necesidades especiales en sus diferentes niveles. Asimismo, cuenta con un profesional en psicología.

La Institución Educativa Nuestra Señora de Copacabana de gestión pública, correspondiente a la UGEL-Puno, ubicada en el jirón Sideral s/n, del centro poblado Santiago de Chejoña en el distrito de Puno, presta servicios a niñas y niños con necesidades especiales con diagnóstico moderado y severo en la modalidad escolarizado en los niveles de educación inicial y educación primaria. La institución cuenta con docentes especializados 
para la atención a la niñez con necesidades especiales en sus dos niveles. No cuenta con un profesional en psicología.

\section{Resultados y análisis}

\subsection{Niveles de aprendizaje en matemática}

Los niveles del aprendizaje en matemática por parte del estudiantado de las instituciones educativas especiales Nuestra Señora de Copacabana (NSC) y Niño Jesús de Praga (NJP) son presentadas en la tabla 2.

Tabla 2

Frecuencia y porcentaje de los estudiantes de las instituciones educativas especiales Nuestra Señora de Copacabana y Niño Jesús de Praga por Nivel de aprendizaje en matemática.

\begin{tabular}{ccccc}
\hline $\begin{array}{c}\text { INSTITUCIÓN } \\
\text { EDUCATIVA }\end{array}$ & \multicolumn{2}{c}{ NIÑO JESÚS DE PRAGA } & \multicolumn{2}{c}{$\begin{array}{c}\text { NUESTRA SEÑORA DE } \\
\text { COPACABANA }\end{array}$} \\
\hline ESCALA & Frecuencia & Porcentaje & Frecuencia & $\begin{array}{c}\text { Porcentaje } \\
\text { Inicio } \\
{[0-10]}\end{array}$ \\
\hline $\begin{array}{c}\text { Proceso } \\
{[11-13]}\end{array}$ & 0 & $0 \%$ & 0 & $0 \%$ \\
\hline $\begin{array}{c}\text { Logro previsto } \\
{[14-17]}\end{array}$ & 18 & $82 \%$ & 15 & $94 \%$ \\
\hline $\begin{array}{c}\text { Logro destacado [18- } \\
20]\end{array}$ & 0 & $18 \%$ & 1 & $6 \%$ \\
\hline TOTAL & 22 & $100 \%$ & 0 & $0 \%$ \\
\hline
\end{tabular}

Fuente: Registro auxiliar de notas de los maestros de las instituciones educativas especiales Nuestra Señora de Copacabana y Niño Jesús de Praga al finalizar el año 2014

Los estudiantes de las instituciones educativas: Niño Jesús de Praga (NJP) y Nuestra Señora de Copacabana (NSC), básicamente se encuentran entre los niveles logro previsto y en proceso (Tabla 2). El $82 \%$ de la comunidad estudiantil de la institución educativa (NJP) y el $94 \%$ de la institución educativa (NSC), se encuentran en proceso, esto significa que los y las estudiantes en este nivel son capaces de establecer relaciones entre la forma y la figura, mide y compara longitudes de los objetos de su entorno en dos y tres dimensiones a través de procedimientos rutinarios y explícitos, no están en la capacidad de asociar lo aprendido con la realidad (PISA, 2012). 
El $18 \%$ de la población estudiantil de la institución educativa (NJP) y el $6 \%$ de la institución educativa (NSC) se encuentran en el nivel logro previsto, evidenciándose que el estudiantado, en este nivel, está en la capacidad de comparar longitudes de los objetos de su entorno en dos y tres dimensiones, y establece relaciones entre la forma y figura geométrica, asociándolos directamente con situaciones del mundo real. Finalmente, estos resultados obtenidos en ambas instituciones guardan relación de similitud (PISA, 2012).

La mayoría de niños y niñas se ubican en el nivel de proceso. Evidentemente, este resultado educativo se debería a la enorme brecha existente entre la edad cronológica y la edad de maduración de la percepción visomotriz para el aprendizaje, esta diferencia es de 9,89 años. Situación que pone en evidencia que no se estarían brindando servicios oportunos a estos discentes debido a que no se están planificando las adaptaciones curriculares ni se están aplicando estrategias conforme a la edad de maduración de la percepción visomotriz. Pinto y Noronha, (2010) sostienen que la provisión oportuna de servicios favorece el éxito escolar.

Esto implica que la adaptación curricular, las estrategias y los grados de dificultad deben ser pertinentes. La programación curricular debe ser planificada con el fin de evitar los fracasos escolares (Bartholomeu, Marín y Sisto, 2005).

Los resultados de esta investigación se alinean con los resultados de Merino (2014, p. 281), en los que reporta que los niveles obtenidos en el área de matemática guardan relación con la edad de madurez, esto significa que para lograr éxitos académicos en la niñez es necesario planificar el currículo acorde con su edad de maduración. Estos resultados también se alinean con la literatura que hace décadas declara que la habilidad visomotora tiene correlatos importantes con los éxitos académicos de estudiantes de los primeros años de escolaridad (Noronha, Marín y Angeli, 2013).

\subsection{Maduración de la percepción visomotriz para el aprendizaje}

Los niveles de maduración de la percepción visomotriz para el aprendizaje evidenciados por los estudiantes con necesidades especiales de las instituciones educativas Niño Jesús de Praga y Nuestra Señora de Copacabana se presentan en la tabla 3. 
Tabla 3

Comparación de edades de maduración para el aprendizaje de los estudiantes de 12,75 años promedio de edad cronológica de las instituciones educativas de educación primaria NJP y NSC en el año 2014

\begin{tabular}{ccccr}
\hline $\begin{array}{c}\text { INSTITUCIÓN } \\
\text { EDUCATIVA }\end{array}$ & \multicolumn{2}{c}{ NIÑO JESÚS DE PRAGA } & \multicolumn{2}{c}{$\begin{array}{c}\text { NUESTRA SEÑORA DE } \\
\text { COPACABANA }\end{array}$} \\
\hline Edad de Maduración & Frecuencia & Porcentaje \% & Frecuencia & Porcentaje \% \\
\hline $\begin{array}{c}\text { [3 años a 4 años] } \\
\text { Pensamiento } \\
\text { simbólico }\end{array}$ & 4 & $18,18 \%$ & 7 & $43,75 \%$ \\
\hline $\begin{array}{c}\text { [4 años a 7 años }] \\
\text { Pensamiento intuitivo, }\end{array}$ & 18 & $81,82 \%$ & 9 & $56,25 \%$ \\
\hline $\begin{array}{c}\text { [7 años a 11 años] } \\
\text { Pensamiento Lógico }\end{array}$ & 0 & $0,00 \%$ & 0 & $0,00 \%$ \\
\hline TOTAL & 22 & $100,00 \%$ & 16 & $100,00 \%$ \\
\hline
\end{tabular}

Fuente: Fichas de registro del test de Bender aplicado a los estudiantes de las instituciones educativas de educación primaria NJP y NSC, 2014.

El $81,82 \%$ de las niñas y de los niños de la institución educativa (NJP) y el 56,25\% de las niñas y de los niños de la institución educativa (NSC) tienen la edad de maduración entre 4 años y 4 años 7 meses para el aprendizaje. Los niños que pertenecen a este intervalo de pensamiento intuitivo se caracterizan por la intensidad en las relaciones sociales, lo cual les permite, junto con la capacidad de lenguaje, el impulso del pensamiento, separar realidades físicas de realidades mentales y establecer diferencias entre estas realidades aunque de manera limitada (PISA, 2012).

El $18,18 \%$ del estudiantado de la institución educativa (NJP) y el $43,75 \%$ de la comunidad estudiantil de la institución educativa (NSC) tienen la edad de maduración entre 2 años y 4 años para el aprendizaje. Los niños y las niñas que pertenecen al intervalo de pensamiento simbólico se caracterizan porque imitan situaciones que ven en la vida real. En este proceso, utilizan al máximo su imaginación, juegan entre lo real y lo imaginario, lo cual les ayuda a crear representaciones mentales que serán de gran ayuda para resolver situaciones de su vida futura (PISA, 2012).

Los resultados referidos a la edad de maduración visomotriz promedio, obtenidos de la aplicación del test de Bender-Koppitz a los estudiantes de las dos instituciones educativas especiales de la ciudad de Puno, son los siguientes: La edad de madurez para el aprendizaje en promedio es de 3,87 años con una desviación estándar de 0,58 años y la edad cronológica es de 12,76 años con una desviación estándar de 3,37 años. Estos resultados evidencian una diferencia importante entre ambas edades, así como muestran que la 
mayoría de la niñez de educación primaria estaría en el nivel inicial. Este hecho demanda que el profesorado, en coordinación con los directivos y padres de familia de las instituciones, debe programar y planificar las adaptaciones curriculares, seleccionar las estrategias y los grados de dificultad de los contenidos temáticos acordes con la edad de maduración.

Rinaldi y Bocato (2012) concluyen que los métodos, técnicas y materiales deben ser planificados en concordancia con la edad de madurez del estudiantado con el fin de brindar servicios educativos adecuados y pertinentes para asegurar el éxito escolar. Del mismo modo, Merino (2011) sostiene la importancia de considerar la edad de madurez en la niñez en etapa escolar, con el propósito de lograr el éxito en el rendimiento académico en la escuela. Según Minedu (2010), el manual de las adaptaciones curriculares indica que el currículo debe ser pertinente a la realidad del estudiante y adaptada a las características que nos proporciona un diagnóstico, por lo que los actores educativos de las instituciones educativas para niños con necesidades especiales deben desarrollar las adaptaciones curriculares, la selección de las estrategias metodológicas y el grado de dificultad de los contenidos temáticos. Por consiguiente, la presente investigación propone que para desarrollar las adaptaciones curriculares, seleccionar las estrategias metodológicas y el grado de dificultad de los contenidos temáticos para los estudiantes con necesidades especiales se debe considerar la edad de maduración visomotriz de los estudiantes, de manera que se garanticen los éxitos académicos (Perrenoud, 2007).

\subsection{Correlación entre los niveles de aprendizaje en matemática y la maduración de la percepción visomotriz.}

Para calcular el coeficiente de correlación, se consideraron a 38 niñas y niños de ambas instituciones educativas especiales de la ciudad de Puno, y las variables de estudio fueron: la primera variable, niveles de aprendizaje en matemática y la segunda variable, edad de maduración de la percepción visomotriz para el aprendizaje, que fue calculada a partir del test de Bender-Kopptiz.

El coeficiente de correlación es $r=0,70$, el cual nos indica que la relación entre ambas variables de estudio es positiva y significativa, (se consideró el valor de $p=0.05$ ) lo que evidencia que el estudiantado con mayor edad de maduración de la percepción visomotriz logra mejores resultados en matemática. Merino (2014, p. 281) reporta en su estudio el coeficiente $r=0,26$ de correlación directa baja entre el rendimiento académico en matemática 
y la edad de madurez, lo que significa que para lograr el éxito en el aprendizaje escolar es necesario considerar la edad de madurez de la niñez en el diseño de sesiones. De tal forma que concuerda con la literatura que hace décadas (década de los noventa) declara que la habilidad visomotora tiene correlatos importantes en los primeros años de la escolaridad (Merino y Allen, 2013). Por otra parte, Rinaldi y Bocato (2012) sostienen que los métodos, técnicas y materiales deben ser planificados en concordancia con la edad de madurez de los niños y las niñas, con el fin de brindar servicios educativos adecuados y pertinentes.

Finalmente, el aporte predictivo de las habilidades de integración visomotora ofrece una visión útil para comprender el aprendizaje de la matemática de la niñez con necesidades especiales, que proponemos sea considerado en el proceso de adaptaciones curriculares. Los niños y las niñas, con una edad de maduración diferente a la edad cronológica establecida para el aprendizaje de la matemática, evidencian una disposición que dificulta el éxito en su aprendizaje. Merino, (2011) y Noronha y Bueri, (2006) concluyen que la disposición para el aprendizaje depende de la edad de maduración visomotora.

\section{Conclusiones}

El coeficiente de correlación $(r=0,70)$ encontrado entre las variables: Niveles de aprendizaje en matemática y la edad de la percepción visomotriz para el aprendizaje es directa y significativa, lo cual significa que el estudiantado con necesidades especiales de mayor edad de maduración de la percepción visomotriz logran mejores resultados de aprendizaje en el área de matemática. El nivel de aprendizaje en matemática de las niñas y niños de la Institución Educativa Niño Jesús de Praga y la Institución Educativa Nuestra Señora de Copacabana se encuentra en proceso. Para mejorar estos resultados se plantea implementar el cálculo de la edad de maduración para el aprendizaje en el proceso de la diversificación curricular, lo cual implica adaptaciones curriculares individuales, selección de estrategias y grado de dificultad de los contenidos temáticos acordes con la edad de maduración de la percepción visomotriz para el aprendizaje, ya que el éxito académico está vinculado directamente con la edad de madurez. 


\section{Referencias}

Allport, Gordon. (1961). Pattern and Growth in personality. New York, USA: Rinehart and Winston press.

Bartholomeu, Daniel, Marín, Fabian y Sisto, Fermino. (2005). Teste de Bender e dificuldades de aprendizagem: Quão válido é o sistema Koppitz. Avaliação Psicológica, 4(1), 13-21.

Bedard, Kelly y Dhuey, Elizabeth. (2006). The Persistence of Early Childhood Maturity: International Evidence of Long-Run Age Effects. The Quarterly Journal of Economics, 121(4), 1437-1472.

Bender, Laureta. (1938). A visual motor Gestalt test and its clinical use. New York, USA: The American Orthopsychiatric Association.

Chan, Pak. (2000). Relationship of the visual motor development and academic performance in young children in Hong Kong assessed in the Bender Gestalt Test. Perceptual and Motor Skills, 90(1), 209-214.

Keller, James. (1955). The use of a Bender-Gestalt maturation level scoring system with mentally handicapped children. Orthopsychiatry 25(3), 563-575.

Koppitz, Elizabeth. (1962). The Bender Gestalt Test witch the Human Figure Drawing Test for Young School Children. Ohio, USA: Columbus.

Koppitz, Elizabeth. (1981). El test gestáltico de Bender. Barcelona, España: Oikos-Tau.

Koppitz, Elizabeth. (1989). O teste gestáltico Bender para crianças. Porto Alegre, Brasil: Artes Médicas.

Merino, César. (2011). Validez de constructo del Sistema Cualitativo de Calificación para el Test Gestáltico de Bender Modificado. Electronic Journal of Research in Educational Psychology, 9(25), 1245-1266.

Merino, Cesar. (2014). Validez incremental del Test Gestáltico de Bender Modificado, en niños que inician el primer grado. Avances en Psicología Latinoamericana, 32(2), 275286.

Merino, César y Allen, Ryan. (2013). Inter-scorer reliability and construct validity in the bender gestalt test (second version). Revista interdisciplinaria, 30(2), 253-264.

Ministerio de Educación de Perú (Minedu). (2010). Manual de adaptaciones curriculares. Lima, Perú: Cimagraf.

Ministerio de Educacion de Perú (Minedu). (2013). Rutas de aprendizaje, Fascículo de matemática. Lima, Perú: Cimagraf.

Noronha, Ana y Bueri, Rosângela. (2006). Koppitz e Bender - Sistema de Pontuação Gradual: comparação entre sistemas de avaliação. Psicología Escolar e Educacional, 10(2), 223-233. 
Noronha, Ana, Marín, Fabián y Angeli, Acácia. (2013). Bender Gestalt Visual-Motor Test Sistema de Pontuação Gradual (B-SPG): A Study With Different Samples. Paidéia, 23(55), 179-185.

Perrenoud, Philippe. (2007). Pedagogía Diferenciada. De las intenciones a la acción. Madrid, España: Editorial Popular.

Pinto, Paula y Noronha, Ana. (2010). Maturidade percepto-motora e sua relação com idade e variáveis contextuais: Um estudo com o Bender (B-SPG). Revista de Psicología, 13(19), 145-155.

Programa Internacional para la Evaluación de Estudiantes (PISA). (2012). El programa Pisa de la OCDE. Que es y para qué sirve. París, Francia: Santillana.

Reynolds, Cecil. (2007). Koppitz developmental scoring system for the Bender gestalt test (KOPPITZ-2). Austin, Texas: Pro-Ed.

Rinaldi, Helena y Boccato, Irai. (2012). Relaçao do teste de Bender (avaliacao Koppitz) com o R-2: testenao verbal de inteligencia para criancas Psicologia. Teoria e Prática, 14(1), 153-167.

Sisto, Fermino, Bartholomeu, Daniel, Rueda, Fabian, Santos, Acácia y Noronha, Ana. (2008). Relações entre o Teste de Bender e matrizes progressivas coloridas de Raven na avaliação da inteligência. Interação em Psicologia, 12(1), 11-19.

Suehiro, Adriana y Santos, Aacácia. (2006). Evidência de validade de critério do BenderSistema de Pontuação. Interação em Psicologia 10(2), 217-224. 